\title{
Texture Enhancement Based on the Savitzky-Golay Fractional Differential Operator
}

\author{
Hamid A. Jalab ${ }^{1}$ and Rabha W. Ibrahim ${ }^{2}$ \\ ${ }^{1}$ Faculty of Computer Science \& Information Technology, University of Malaya, 50603 Kuala Lumpur, Malaysia \\ ${ }^{2}$ Institute of Mathematical Sciences, University of Malaya, 50603 Kuala Lumpur, Malaysia \\ Correspondence should be addressed to Rabha W. Ibrahim; rabhaibrahim@yahoo.com
}

Received 13 August 2012; Revised 5 January 2013; Accepted 5 January 2013

Academic Editor: José Tenreiro Machado

Copyright (c) 2013 H. A. Jalab and R. W. Ibrahim. This is an open access article distributed under the Creative Commons Attribution License, which permits unrestricted use, distribution, and reproduction in any medium, provided the original work is properly cited.

\begin{abstract}
Texture enhancement for digital images is the most important technique in image processing. The purpose of this paper is to design a texture enhancement technique using fractional order Savitzky-Golay differentiator, which leads to generalizing the Savitzky-Golay filter in the sense of the Srivastava-Owa fractional operators. By employing this generalized fractional filter, texture enhancement is introduced. Consequently, it calculates the generalized fractional order derivative of the given image using the sliding weight window over the image. Experimental results show that the operator can extract more subtle information and make the edges more prominent. In general, the capability of the generalized fractional differential will be high because it is sensitive to the subtle fluctuations of values of pixels.
\end{abstract}

\section{Introduction}

Texture is an important feature of natural images; hence, a variety of image texture applications has been intensively studied by many researchers [1]. Image texture is defined as a function of the spatial variation in pixel intensities (gray values). Smith and Chang [2] have defined texture as visual patterns, which have properties of homogeneity and not resulting from only a single color or intensity.

In the image, texture features capture information about repeating patterns. Texture analysis can be classified into three models: structural, statistical, and signal theoretic methods [3]. Therefore, the analysis of texture parameters is a useful approach for increasing the information accessible from images. In texture enhancement technique, which is based on mask operation, each pixel is modified according to value of the neighbourhood around the pixel of interest. One important aspect of an image, which enables us to perform this, is the notion of frequencies. Fundamentally, the frequencies of an image are the amount, by which the gray values change with distance. High-frequency components are characterized by huge changes in gray values over small distances; examples of high frequency components are edges and noise. On the other hand, low-frequency components are parts of the image, which are characterized by little change in the gray values [4]. Fractional differential mask can further preserve the low-frequency contour feature in those smooth areas, and nonlinearly keep the high-frequency marginal feature in those areas, where the gray-level changes heavily, and also enhances texture details in those areas, where the gray-level does not change evidently.

Fractional integration and fractional differentiation are generalizations of notions of integer-order integration and differentiation and include $n$th derivatives and $n$-fold integrals as particular cases $[5,6]$. Many applications of fractional calculus in physics have replaced the time derivative in an evolution equation with a derivative of fractional order [7-11]. Fractional calculus has been applied to a variety of physical phenomena, including anomalous diffusion, transmission line theory, problems involving oscillations, nanoplasmonics, solid mechanics, astrophysics, and viscoelasticity. Currently, fractional calculus (integral and differential operators) is heavily used in control design $[12,13]$, Furthermore, in image processing [14-19], all results that are based on the fractional 
calculus operators (differential and integral) show that this method not only is effective, but also has good immunity.

The digital fractional order differentiator is an important topic in fractional calculus that can estimate the fractional order derivative of any given digital signals, without known function. The Savitzky-Golay filter is a simplified digital differentiator that is implemented by a local polynomial regression technique $[20,21]$. Now, Savitzky-Golay digital differentiator has been one of the most popular numerical differentiation methods, due to its high computing speed and strong antinoise ability.

Recently, the interest in using texture enhancement technique based on mask operation has grown in the field of image processing. $\mathrm{Pu}$ and Zhou [22] have implemented multiscale texture segmentation by fractional differential. They have proposed two fractional differential masks and presented the structures and parameters of each mask, respectively. Then they have discussed the multi-scale texture segmentation based on the fractional mask. $\mathrm{Pu}$ [23] has proposed a fractional calculus approach to enhance the texture of digital image. He has found that the textural detail enhancing capability of fractional derivative-based texture operator is much better than integer derivative. Zhang et al. [24] have used the fractional differential masks based on the classical Riemann-Liouville definition. They have concluded that the fractional order between 1 and 2 can enhance the texture and edges in multiscale, by controlling the fractional order.

In this paper, we have used a generalized fractional differential based on the generalized Savitzky-Golay filter in sense of Srivastava-Owa fractional operators for image texture enhancement. The Savitzky-Golay filter has become a powerful signal and image processing tool, which has found application in many scientific areas. Moreover, the SavitzkyGolay filter method is considered to be a good approach in image texture enhancement, which is used as an alternative to classical techniques. The rest of the paper is organized as follows: Sections 2 and 3 explain the fractional calculus and the generalized fractional integral operator, respectively, Section 4 describes the construction of fractional differential Savitzky-Golay filter, Section 5 elucidates the experimental results, and Section 6 concludes the paper.

\section{Fractional Calculus}

The idea of the fractional calculus (i.e., calculus of integrals and derivatives of any arbitrary real or complex order) was found over 300 years ago. Abel in 1823 scrutinized the generalized tautochrone problem and for the first time applied fractional calculus techniques in a physical problem.

2.1. The Riemann-Liouville Operators. The RiemannLiouville fractional derivative strongly poses the physical interpretation of the initial conditions required for the initial value problems involving fractional differential equations. Moreover, this operator possesses advantages of fast convergence, high stability, and higher accuracy to derive different types of numerical algorithms [6].
The fractional (arbitrary) order integral of the function $f$ of order $\alpha>0$ is defined by

$$
I_{a}^{\alpha} f(t)=\int_{a}^{t} \frac{(t-\tau)^{\alpha-1}}{\Gamma(\alpha)} f(\tau) d \tau
$$

When $a=0$, we write $I_{a}^{\alpha} f(t)=f(t) * \phi_{\alpha}(t)$, where $(*)$ denoted the convolution product, $\phi_{\alpha}(t)=t^{\alpha-1} / \Gamma(\alpha), t>0$ and $\phi_{\alpha}(t)=0, t \leq 0$, and $\phi_{\alpha} \rightarrow \delta(t)$ as $\alpha \rightarrow 0$, where $\delta(t)$ is the delta function.

The fractional (arbitrary) order derivative of the function $f$ of order $0 \leq \alpha<1$ is defined by

$$
D_{a}^{\alpha} f(t)=\frac{d}{d t} \int_{a}^{t} \frac{(t-\tau)^{-\alpha}}{\Gamma(1-\alpha)} f(\tau) d \tau=\frac{d}{d t} I_{a}^{1-\alpha} f(t) .
$$

When $a=0$, we have

$$
\begin{gathered}
D^{\alpha} t^{\mu}=\frac{\Gamma(\mu+1)}{\Gamma(\mu-\alpha+1)} t^{\mu-\alpha}, \quad \mu>-1, \quad 0<\alpha<1, \\
I^{\alpha} t^{\mu}=\frac{\Gamma(\mu+1)}{\Gamma(\mu+\alpha+1)} t^{\mu+\alpha}, \quad \mu>-1, \alpha>0 .
\end{gathered}
$$

The Caputo fractional derivative of order $\mu>0$ is defined, for a smooth function $f$, by

$$
{ }^{c} D_{a}^{\mu} f(t)=\int_{a}^{t} \frac{(t-\tau)^{n-\mu-1}}{\Gamma(n-\mu)} f^{(n)}(\tau) d \tau,
$$

where $n=[\mu]+1$ (the notation $[\mu]$ stands for the largest integer not greater than $\mu$ ). Note that there is a relationship between the Riemann-Liouville differential operator and the Caputo operator:

$$
D_{a}^{\mu} f(t)={ }^{c} D_{a}^{\mu} f(t)+\frac{1}{\Gamma(1-\mu)} \frac{f(a)}{(t-a)^{\mu}},
$$

and they are equivalent in a physical problem (i.e., a problem which specifies the initial conditions).

2.2. The Srivastava-Owa Operators. In [25], Srivastava and Owa defined and studied fractional operators (derivative and integral) in the complex $z$-plane $\mathbb{C}$ for analytic functions.

The fractional derivative of order $\beta$ is defined, for a function $f(z)$ by

$$
D_{z}^{\beta} f(z):=\frac{1}{\Gamma(1-\beta)} \frac{d}{d z} \int_{0}^{z} \frac{f(\zeta)}{(z-\zeta)^{\beta}} d \zeta, \quad 0 \leq \beta<1,
$$

where the function $f(z)$ is analytic in simply-connected region of the complex $z$-plane $\mathbb{C}$ containing the origin and the multiplicity of $(z-\zeta)^{-\beta}$ is removed by requiring $\log (z-\zeta)$ to be real when $(z-\zeta)>0$. Furthermore, for $n \leq \beta<n+1$, the fractional differential operator is defined as

$$
D_{z}^{\beta} f(z)=\frac{d^{n}}{d z^{n}} D_{z}^{\beta-n} f(z), \quad n \in \mathbb{N} .
$$


The fractional integral of order $\beta$ is defined, for a function $f(z)$, by

$$
I_{z}^{\beta} f(z):=\frac{1}{\Gamma(\beta)} \int_{0}^{z} f(\zeta)(z-\zeta)^{\beta-1} d \zeta, \quad \beta>0,
$$

where the function $f(z)$ is analytic in simply connected region of the complex $z$-plane $(\mathbb{C})$ containing the origin and the multiplicity of $(z-\zeta)^{\beta-1}$ is removed by requiring $\log (z-\zeta)$ to be real when $(z-\zeta)>0$ :

$$
\begin{gathered}
D_{z}^{\beta} z^{\mu}=\frac{\Gamma(\mu+1)}{\Gamma(\mu-\beta+1)} z^{\mu-\beta}, \quad \mu>-1,0 \leq \beta<1 \\
I_{z}^{\beta} z^{\mu}=\frac{\Gamma(\mu+1)}{\Gamma(\mu+\beta+1)} z^{\mu+\beta}, \quad \mu>-1, \beta>0 .
\end{gathered}
$$

Note that the real case of the Srivastava-Owa operators is equivalence to the Riemann-Liouville operators.

\section{Generalized Fractional Integral Operator}

This section briefly describes the mathematical background for the fractional integral operator that has been used by the proposed algorithm. The usual way of representing the fractional derivatives is by the Riemann-Liouville formula $D_{t}^{\alpha}$. Another way to represent the fractional derivatives is by the Grünwald-Letnikov formula [23]. The discrete approximations derived from the Grünwald-Letnikov fractional derivatives present some limitations, such as the following[26]:

(i) they frequently originate unstable numerical methods;

(ii) the order of accuracy of such approaches is never higher than one.

To implement the generalized fractional integral method, Ibrahim in [27] has imposed a formula for the generalized fractional integral. Consider, for natural $n \in \mathbb{N}=\{1,2, \ldots\}$ and real $\mu$, the $n$-fold integral of the form

$$
I_{z}^{\alpha, \mu} f(z)=\int_{0}^{z} \zeta_{1}^{\mu} d \zeta_{1} \int_{0}^{\zeta_{1}} \zeta_{2}^{\mu} d \zeta_{2} \cdots \int_{0}^{\zeta_{n-1}} \zeta_{n}^{\mu} f\left(\zeta_{n}\right) d \zeta_{n}
$$

Applying the Cauchy formula for iterated integrals implies

$$
\begin{aligned}
\int_{0}^{z} \zeta_{1}^{\mu} d \zeta_{1} \int_{0}^{\zeta_{1}} \zeta^{\mu} f(\zeta) d \zeta & =\int_{0}^{z} \zeta^{\mu} f(\zeta) d \zeta \int_{\zeta}^{z} \zeta_{1}^{\mu} d \zeta_{1} \\
& =\frac{1}{\mu+1} \int_{0}^{z}\left(z^{\mu+1}-\zeta^{\mu+1}\right) \zeta^{\mu} f(\zeta) d \zeta .
\end{aligned}
$$

Repeating the above step $n-1$ times we obtain

$$
\begin{aligned}
& \int_{0}^{z} \zeta_{1}^{\mu} d \zeta_{1} \int_{0}^{\zeta_{1}} \zeta_{2}^{\mu} d \zeta_{2} \cdots \int_{0}^{\zeta_{n-1}} \zeta_{n}^{\mu} f\left(\zeta_{n}\right) d \zeta_{n} \\
& =\frac{(\mu+1)^{1-n}}{(n-1) !} \int_{0}^{z}\left(z^{\mu+1}-\zeta^{\mu+1}\right)^{n-1} \zeta^{\mu} f(\zeta) d \zeta
\end{aligned}
$$

which yields the fractional operator type

$$
I_{z}^{\alpha, \mu} f(z)=\frac{(\mu+1)^{1-\alpha}}{\Gamma(\alpha)} \int_{0}^{z}\left(z^{\mu+1}-\zeta^{\mu+1}\right)^{\alpha-1} \zeta^{\mu} f(\zeta) d \zeta
$$

where $\alpha$ and $\mu \neq-1$ are real numbers and the function $f(z)$ is analytic in the simply connected region of the complex $z$ plane $\mathbb{C}$ containing the origin, and the multiplicity of $\left(z^{\mu+1}-\right.$ $\left.\zeta^{\mu+1}\right)^{-\alpha}$ is removed by requiring $\log \left(z^{\mu+1}-\zeta^{\mu+1}\right)$ to be real when $\left(z^{\mu+1}-\zeta^{\mu+1}\right)>0$. When $\mu=0$, we arrive at the standard Srivastava-Owa fractional integral, which is used to define the Srivastava-Owa fractional derivatives.

Corresponding to the generalized fractional integrals (13), we define the generalized differential operator of order $\alpha$ by

$$
D_{z}^{\alpha, \mu} f(z):=\frac{(\mu+1)^{\alpha}}{\Gamma(1-\alpha)} \frac{d}{d z} \int_{0}^{z} \frac{\zeta^{\mu} f(\zeta)}{\left(z^{\mu+1}-\zeta^{\mu+1}\right)^{\alpha}} d \zeta, \quad 0<\alpha \leq 1,
$$

where the function $f(z)$ is analytic in the simply connected region of the complex $z$-plane $\mathbb{C}$ containing the origin and the multiplicity of $\left(z^{\mu+1}-\zeta^{\mu+1}\right)^{-\alpha}$ is removed by requiring $\log \left(z^{\mu+1}-\zeta^{\mu+1}\right)$ to be real when $\left(z^{\mu+1}-\zeta^{\mu+1}\right)>0$.

Proposition 1 (see [27]). The generalized derivative of the function $f(z)=z^{v}, v \in \mathbb{R}$ is given by the following:

$$
D_{z}^{\alpha, \mu} f(z)=\frac{(\mu+1)^{\alpha-1} \Gamma(\nu /(\mu+1)+1)}{\Gamma(\nu /(\mu+1)+1-\alpha)} z^{(1-\alpha)(\mu+1)+v-1},
$$

which is later used to compute the coefficient matrix $W^{(\alpha, \mu)}$.

\section{Construction of the Fractional Differential Savitzky-Golay Filter}

The Savitzky-Golay filter has been introduced for computing the numerical derivatives and is also called a digital smoothing polynomial filter. The Savitzky-Golay method is often used to preserve higher moments in the data, thus reducing the distortion of essential features of the data.

In this section, we will generalize this filter for calculating the fraction derivatives which will be utilized by the proposed algorithm.

Assume a uniformly sampled signal, our aim is to estimate its $n$th order derivative using $I$-point filtering window and an n-degree polynomial [21]:

$$
f_{n}(i)=\sum_{k=0}^{n} a_{k} i^{k}
$$

which is used to fit the given signal $i=1,2, \ldots, I$. In matrix notation, (16) is reduced to the system

$$
Y=X A+\varepsilon,
$$


where $\varepsilon$ is the estimate error, $A$ is the $n+1 \times 1$ coefficient matrix and $X$ is the $I \times(n+1)$ Vandermonde matrix defined by

$$
X=\left(\begin{array}{cccc}
1 & 1^{1} & \cdots & 1^{n} \\
1 & 2^{1} & \cdots & 2^{n} \\
\vdots & \vdots & \cdots & \vdots \\
1 & I^{1} & \cdots & I^{n}
\end{array}\right)
$$

The coefficients of the best-fit polynomial can be obtained by minimizing the sum of the squared errors between the actual data and fitting points. Thus,

$$
B=\left(X^{T} X\right)^{-1} X^{T} Y
$$

implies

$$
\widehat{Y}=W Y,
$$

where $W$ denotes the moving window's coefficients matrix. Consequently, the $n$th order derivative can be estimate by

$$
\widehat{Y}^{(d n)}=W^{(d n)} Y
$$

Now, in view of Proposition 1, we have

$$
\widehat{Y}^{(\alpha, \mu)}=W^{(\alpha, \mu)} Y=A\left(X^{T} X\right)^{-1} X^{T} Y,
$$

where

$$
\begin{aligned}
A= & {\left[\frac{(\mu+1)^{\alpha-1}}{\Gamma(1-\alpha)} i^{(1-\alpha)(\mu+1)-1},\right.} \\
& \frac{(\mu+1)^{\alpha-1} \Gamma(1 /(\mu+1)+1)}{\Gamma(1 /(\mu+1)+1-\alpha)} i^{(1-\alpha)(\mu+1)}, \ldots, \\
& \left.\frac{(\mu+1)^{\alpha-1} \Gamma(n /(\mu+1)+1)}{\Gamma(n /(\mu+1)+1-\alpha)} i^{(1-\alpha)(\mu+1)+n-1}\right] .
\end{aligned}
$$

Note that when $\mu=0$, we have the Riemann-Liouville differential operator. Moreover, when $I=n+1$, the Vandermonde matrix $X$ is a square matrix. The purpose of the Savitzky-Golay filter is to estimate $W^{(\alpha, \mu)}$, which can be used to calculate the $n$th order derivative of any given signal [21]. The coefficient matrix $W^{(\alpha, \mu)}$ can be computed by

$$
W^{(\alpha, \mu)}=A X^{-1}
$$

where

$$
X=\left(\begin{array}{cccc}
1 & 1^{1} & \cdots & 1^{n} \\
1 & (1+\phi)^{1} & \cdots & (1+\phi)^{n} \\
1 & (1+2 \phi)^{1} & \cdots & (1+2 \phi)^{n} \\
\vdots & \vdots & \cdots & \vdots \\
1 & I^{1} & \cdots & I^{n}
\end{array}\right)
$$

where $\phi$ is the sampling interval.
TABLE 1: Moving window of fractional differential weights with $3 \times$ 3 dimension.

\begin{tabular}{ccc}
\hline 1 & $1^{1}$ & $1^{2}$ \\
1 & $(1+\phi)^{1}$ & $(1+\phi)^{2}$ \\
1 & $I^{1}$ & $I^{2}$ \\
\hline
\end{tabular}

TABLE 2: Moving window of fractional differential weights with $3 \times$ 3 dimension for $\varphi=1, \alpha=0.4$, and $\mu=0.5$.

\begin{tabular}{lcc}
\hline 0.2268 & 0.3057 & -0.0060 \\
-0.8687 & 1.2983 & 0.0420 \\
-1.0277 & 1.2742 & 0.2016 \\
\hline
\end{tabular}

TABLE 3: Moving window of fractional differential weights with $3 \times$ 3 dimension for $\varphi=1, \alpha=0.5$, and $\mu=0.5$.

\begin{tabular}{lcc}
\hline 0.0732 & 0.3951 & -0.0076 \\
-0.8192 & 1.126 & 0.0416 \\
-0.7999 & 0.9307 & 0.1772 \\
\hline
\end{tabular}

The matrix $X$ can be assumed as the formula of computation time; therefore, the generalized Savitzky-Golay filter can be viewed as the generalization of the differentiator. However, digital image is a function of two variables, so, we can generalize these definitions to include both the $x$ and $y$ values.

The mask is designed into $r \times r$ size matrix $M$ which has $r$ layers ( $r$ is odd). The window's size can be an arbitrary odd number, and a larger window can improve the accuracy of fractional differential, but increases the computational time. Therefore, we proceed to use moving window $W^{(\alpha, \mu)}$ with $3 \times 3$ size as shown in Table 1.

The fractional differential operator can enhance edges and contours as well as reserve the texture details. The nine values output of each fractional differential window $h(x, y)$ is performed by sliding the mask window $w(s, t)$ over the image $f$. Generally one can start at the top left corner of the image block through all the pixels, where the fractional differential mask fits entirely within the boundaries of the image. The output of each image block is nine values, which represent the texture information in each image block, that takes the following formula:

$$
h(x, y)=w(s, t) f(x+s, y+t),
$$

where $f$ is the value of an image pixel and $w$ is the value of filter mask.

\section{Experimental Results and Discussion}

The reason of this experiment is to validate the correctness of the proposed algorithm.

Performance tests for the algorithm proposed by this paper were implemented using Matlab 2010a on Intel (R) Core i7 at 2.2 GHz, 4 GB DDR3 Memory, and system type 64-bit, Window 7. The computation time per image differs for each image and depends mainly on the window's size as well as image size. 
TABLE 4: Performance evaluation of the proposed algorithm compared to four statistical measures for image (a).

\begin{tabular}{|c|c|c|c|c|}
\hline Images & Entropy & Homogeneity & Contrast & Energy \\
\hline Original image (a) & 0.036 & 0.999 & 0.025 & 0.998 \\
\hline Proposed algorithm with $(\mu=0.5, \alpha=0.4)$ & 0.224 & 0.949 & 2.767 & 0.881 \\
\hline Proposed algorithm with $(\mu=0.5, \alpha=0.5)$ & 0.260 & 0.941 & 3.219 & 0.860 \\
\hline
\end{tabular}

TABLE 5: Performance evaluation of the proposed algorithm compared to four statistical measures for image (b).

\begin{tabular}{|c|c|c|c|c|}
\hline Images & Entropy & Homogeneity & Contrast & Energy \\
\hline Original image (b) & 0 & 1 & 0 & 1 \\
\hline Proposed algorithm with $(\mu=0.5, \alpha=0.4)$ & 0.017 & 0.997 & 0.112 & 0.994 \\
\hline Proposed algorithm with $(\mu=0.5, \alpha=0.5)$ & 0.032 & 0.995 & 0.214 & 0.989 \\
\hline
\end{tabular}

The proposed texture features enhancement algorithm includes the following steps:

(i) read the original gray-scale image;

(ii) set the value of $n$ and $I(\geq 1)$;

(iii) set the value of the image sampling interval $\phi$;

(iv) set the values of the fractional power parameters $(\alpha, \mu \in(0,1))$;

(v) compute Savitzky-Golay moving window $W^{(\alpha, \mu)}$ as in (24);

(vi) compute the Vandermonde matrix $X$ as in (25);

(vii) apply the Savitzky-Golay fractional differential mask with the corresponding image pixels by sliding the window over the image.

By varying both the fractional powers $\alpha$ and $\mu$, keeping $I$, and $n$ fixed $(I=9, n=2)$, the elements of the SavitzkyGolay moving window $W^{(\alpha, \mu)}$ have been computed as shown in Tables 2 and 3.

Tables 2 and 3. show the coefficients of the fractional differential moving window for different values of $\alpha$. All coefficient values are not equal to zero, which implies that the magnitude response of Savitzky-Golay filter is not also zero in the image region. This will likely improve the texture detail. However, the qualities of texture is defined by the spatial distribution of gray values for this reason, we have used gray-scale images for testing, which are shown in Figures 1(a) and 1(b).

In order to illustrate the efficiency of the proposed algorithm in Figure 2, we have presented an illustration of the obtained results for the texture enhancement of the original images of Figure 1.

The proposed enhancement algorithm shows good enhancement performance for both, testing images by different degrees of fractional power values $\alpha$ and $\mu$ which are experimentally fixed at $\alpha=0.4,0.5$ and $\mu=0.5$, and the value of the image sampling interval $\varphi=1$. It is seen that, the proposed enhancement algorithm using fractional differential masks, can extract more texture information and sharpen edges more efficiently. The eye's qualitative analysis of the proposed algorithm acts as one of the important parameters to judge its performance.
Other metrics used to judge the algorithm performance are the statistical measures. In this paper, among the statistical features, the following second-order statistics are used as texture features in representing images. The gray-level cooccurrence matrix (GLCM) is a statistical method used to describe textures in an image, by modeling texture as a two-dimensional gray level variation [28]. Four statistical measures are extracted to evaluate the images texture enhancement; these are entropy, homogeneity, contrast, and energy.

(1) Entropy measures the amount of information, and the larger value of entropy is the greater amount of information carried by image, but inversely correlated to energy. Entropy feature of gray-scale cooccurrence matrix is one of the features having the best discriminatory power, which is given in following equation:

$$
\text { Entropy }=\sum_{i, j} p(i, j) \log p(i, j) \text {, }
$$

where $p(i, j)$ is the probability for gray-scale $i$ and $j$ and occurs at two pixels.

(2) Homogeneity measures the closeness of the distribution of elements in the GLCM to the GLCM diagonal:

$$
\text { Homogeneity }=\sum_{i, j} \frac{p(i, j)}{1+|i-j|} \text {. }
$$

(3) Contrast measures the intensity contrast between a pixel and its neighbour over the whole image:

$$
\text { Contrast }=\sum_{i, j} p(i-j)^{2} \text {. }
$$

(4) Energy measures the sum of squared elements in the gray-level cooccurrence matrix (GLCM):

$$
\text { Energy }=\sum_{i, j} p(i, j)^{2} .
$$

Figure 2 shows the results of the proposed enhancement algorithm for $\mu=0.5$ with (a) $\alpha=0.4$, and (b) $\alpha=0.5$. 


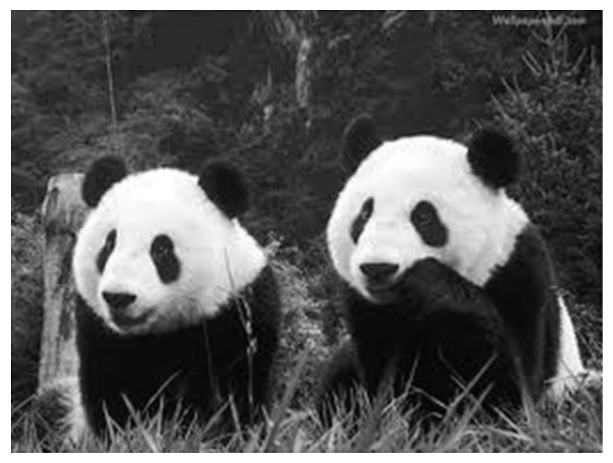

(a)

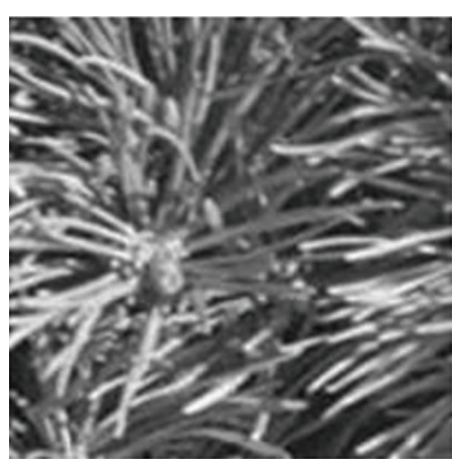

(b)

FIgURE 1: Original images.

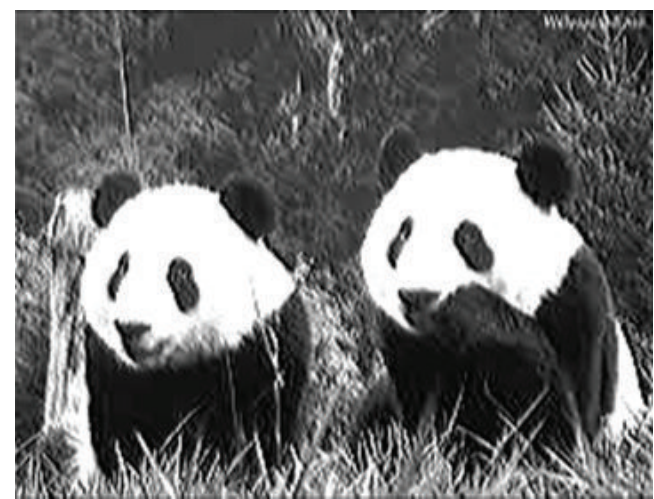

(a)

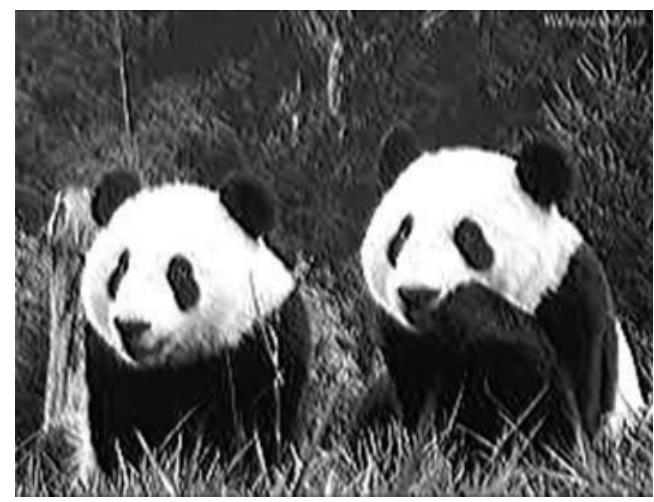

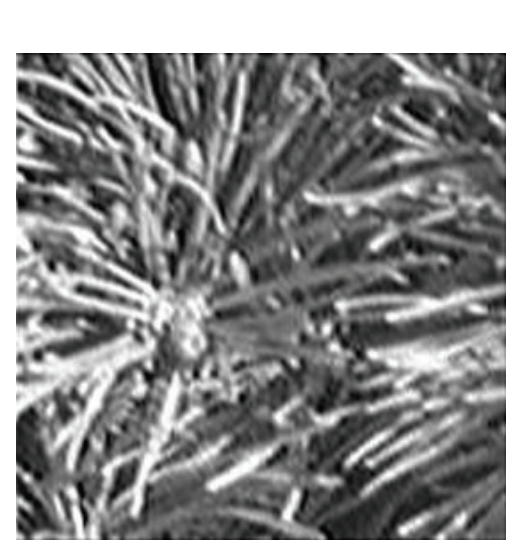

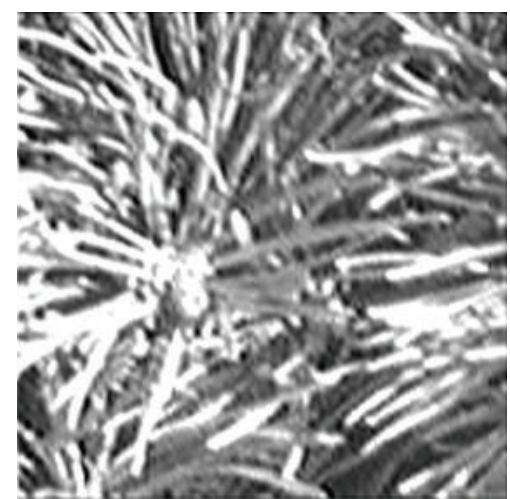

(b)

Figure 2: The results of the proposed algorithm for $\mu=0.5$ and; (a) $\alpha=0.4$, (b) $\alpha=0.5$.

The variation of the image texture is observed when $\alpha$ is increased from 0.4 to 0.5 . So, the selection of differential order is important.

Tables 4 and 5 and Figures 3 and 4 show the performance evaluation of the proposed algorithm for image (a) and image (b) according to those four statistical measures of gray-level cooccurrence matrix (GLCM). It can be clearly seen that there has been a large increase in the value of entropy, which means the greater amount of information is carried by image due to texture enhancement. The entropy values for image (a) climbed to approximately 0.224 for $\alpha=0.4$ and to 0.26 for 


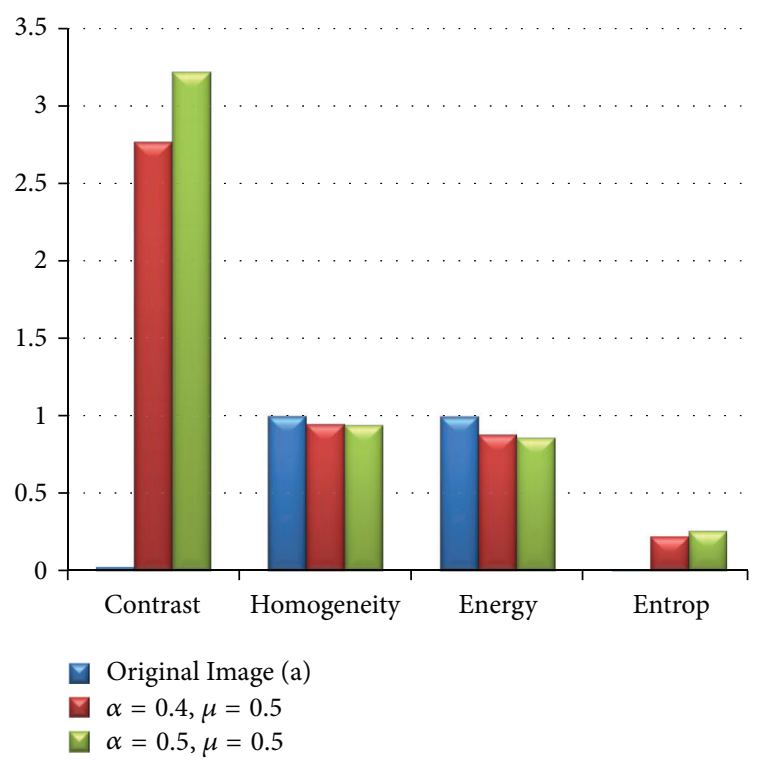

FIGURE 3: The evaluation performance of the proposed algorithm compared to four statistical measures for image (a).

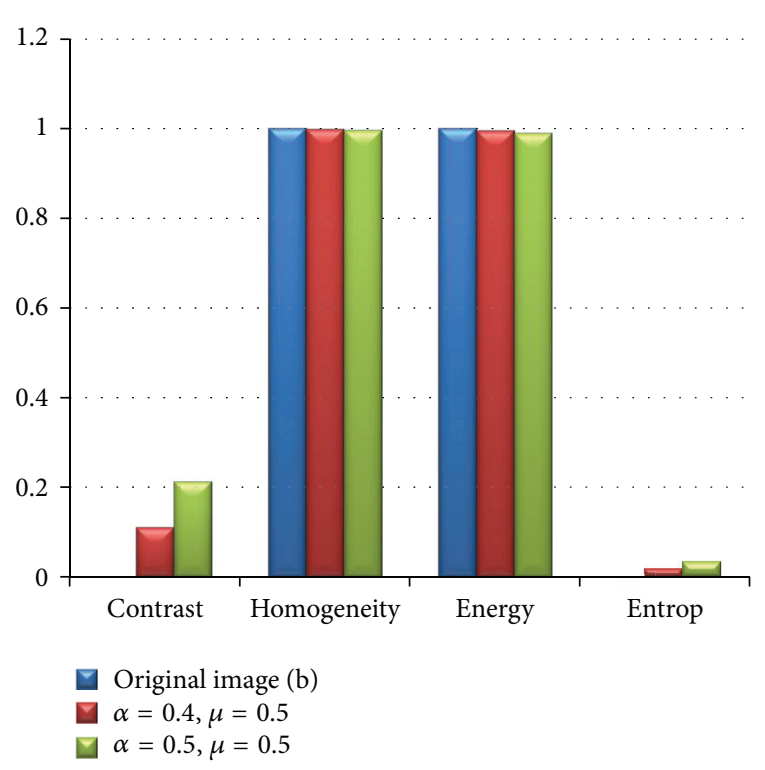

FIGURE 4: The evaluation performance of the proposed algorithm compared to four statistical measures for image (b).

statistical measures makes the proposed algorithm capable to control the degree of texture enhancement of the image by controlling the fractional order parameters $\alpha, \mu$ and $\varphi$.

\section{Conclusion}

In this paper, a texture enhancement technique using fractional order Savitzky-Golay differentiator, which leads to generalize Savitzky-Golay filter in sense of Srivastava-Owa fractional operators, have been introduced. The new algorithm presented in this paper can control the degree of texture enhancement of the image with the fractional power values. The new approach can control the degree of texture enhancement of the image with fractional order of the parameters $\alpha, \mu$, and $\varphi$. However, the technique is by no means limited only to images, instead, it can be applied in the setting of different image applications, taking into consideration the limitations of each imaging method. Furthermore, our goal is to keep away from the effect of the noise that caused in the texture enhancement of the image and to control the degree of texture enhancement of the image with the filter mask parameters. The experiment results had demonstrated the efficacy of this algorithm according to the metrics used to judge the algorithm performance.

\section{Acknowledgments}

The authors would like to thank the reviewers for their comments on earlier versions of this paper. This research has been funded by university of Malaya, under Grant no. UMRG 104-12ICT.

\section{References}

[1] H. A. Jalab, "Image retrieval system based on color layout descriptor and Gabor filters," in Proceedings of the IEEE Conference on Open Systems (ICOS '11), pp. 32-36, 2011.

[2] J. R. Smith and S. F. Chang, "Automated binary texture feature sets for image retrieval," in Proceedings of the IEEE International Conference on Acoustics, Speech, and Signal Processing (ICASSP '96), pp. 2239-2242, May 1996.

[3] N. Bouguila and R. I. Hammoud, "Color texture classification by a discrete statistical model and feature selection," in Proceedings of the IEEE International Conference on Image Processing (ICIP '09), pp. 1381-1384, November 2009.

[4] A. McAndrew, "An introduction to digital image processing with matlab notes for SCM2511 image processing," in School of Computer Science and Mathematics, pp. 1-264, Victoria University of Technology, 2004.

[5] S. G. Samko, A. A. Kilbas, and O. I. Marichev, Fractional Integrals and Derivatives, Gordon and Breach Science Publishers, Yverdon, Switzerland, 1993.

[6] K. S. Miller and B. Ross, An Introduction to the Fractional Calculus and Fractional Differential Equations, A Wiley-Interscience Publication, John Wiley \& Sons, New York, NY, USA, 1993.

[7] A. Oustaloup, F. Levron, B. Mathieu, and F. M. Nanot, "Frequency-band complex noninteger differentiator: characterization and synthesis," IEEE Transactions on Circuits and Systems I, vol. 47, no. 1, pp. 25-39, 2000.

[8] R. Malti, M. Aoun, F. Levron, and A. Oustaloup, "Analytical computation of the $\mathrm{H}_{2}$-norm of fractional commensurate transfer functions," Automatica, vol. 47, no. 11, pp. 2425-2432, 2011.

[9] R. Malti, X. Moreau, F. Khemane, and A. Oustaloup, "Stability and resonance conditions of elementary fractional transfer functions," Automatica, vol. 47, no. 11, pp. 2462-2467, 2011.

[10] S. Victor, P. Melchior, and A. Oustaloup, "Robust path tracking using flatness for fractional linear MIMO systems: a thermal 
application," Computers \& Mathematics with Applications, vol. 59, no. 5, pp. 1667-1678, 2010.

[11] L. Sommacal, P. Melchior, A. Dossat et al., "Improvement of the muscle fractional multimodel for low-rate stimulation," Biomedical Signal Processing and Control, vol. 2, no. 3, pp. 226233, 2007.

[12] D. N. Gruel, P. Lanusse, and A. Oustaloup, "Robust control design for multivariable plants with time-delays," Chemical Engineering Journal, vol. 146, no. 3, pp. 414-427, 2009.

[13] P. Lanusse, A. Oustaloup, and J. Sabatier, "Robust design of an anti-windup compensated 3rd-generation CRONE controller," in Advances in Fractional Calculus, pp. 527-542, Springer, Dordrecht, The Netherlands, 2007.

[14] J. Hu, Y. Pu, and J. Zhou, "A novel image denoising algorithm based on riemann-liouville definition," Journal of Computers, vol. 6, no. 7, pp. 1332-1338, 2011.

[15] H. A. Jalab and R. W. Ibrahim, "Denoising algorithm based on generalized fractional integral operator with two parameters," Discrete Dynamics in Nature and Society, vol. 2012, Article ID 529849, 14 pages, 2012.

[16] H. Jalab and R. Ibrahim, "Texture feature extraction based on fractional mask convolution with cesáro means for contentbased image retrieval," in Proceedings of the 12th Pacific Rim Conference on Artificial Intelligence, pp. 170-179, 2012.

[17] Y. Liu, Y. Pu, and J. Zhou, "Design of image denoising filter based on fractional integral," Journal of Computational Information Systems, vol. 6, no. 9, pp. 2839-2847, 2010.

[18] J. Hu, Y. Pu, and J. Zhou, "Fractional integral denoising algorithm and implementation of fractional integral filter," Journal of Computational Information Systems, vol. 7, no. 3, pp. 729-736, 2011.

[19] B. Mathieu, P. Melchior, A. Oustaloup, and C. Ceyral, "Fractional differentiation for edge detection," Signal Processing, vol. 83, no. 11, pp. 2421-2432, 2003.

[20] R. W. Schafer, "What is a savitzky-golay filter?" IEEE Signal Processing Magazine, vol. 28, no. 4, pp. 111-117, 2011.

[21] D. Chen, D. Xue, and F. Pan, "Digital fractional order SavitzkyGolay differentiator," in IEEE Transactions on Circuits and Systems II, vol. 58, pp. 758-762, 2011.

[22] Y.-F. Pu and J.-L. Zhou, "A novel approach for multi-scale texture segmentation based on fractional differential," International Journal of Computer Mathematics, vol. 88, no. 1, pp. 58-78, 2011.

[23] Y. Pu, "Fractional calculus approach to texture of digital image," in Proceedings of the 8th International Conference on Signal Processing (ICSP '06), November 2006.

[24] Y. Zhang, Y. Pu, and J. Zhou, "Construction of fractional differential masks based on Riemann-Liouville definition," Journal of Computational Information Systems, vol. 6, no. 10, pp. 3191-3200, 2010.

[25] H. M. Srivastava and S. Owa, Univalent Functions, Fractional Calculus, and Their Applications, Ellis Horwood, Chichester, UK, 1989.

[26] E. Sousa, "How to approximate the fractional derivative of order $1<\alpha \leq 2$," International Journal of Bifurcation and Chaos in Applied Sciences and Engineering, vol. 22, no. 4, 13 pages, 2012.

[27] R. W. Ibrahim, "On generalized Srivastava-Owa fractional operators in the unit disk," Advances in Difference Equations, vol. 2011, pp. 1-10, 2011.

[28] S. Selvarajah and S. Kodituwakku, "Analysis and comparison of texture features for content based image retrieval," International Journal of Latest Trends in Computing, vol. 108, 2011. 


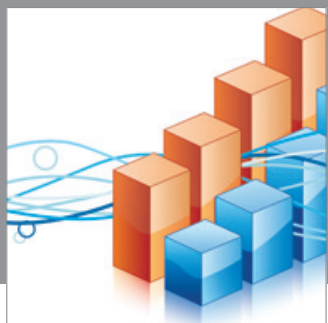

Advances in

Operations Research

mansans

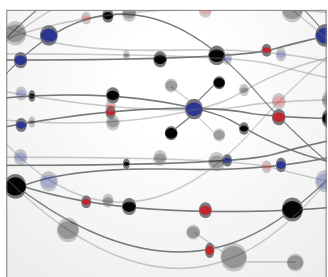

The Scientific World Journal
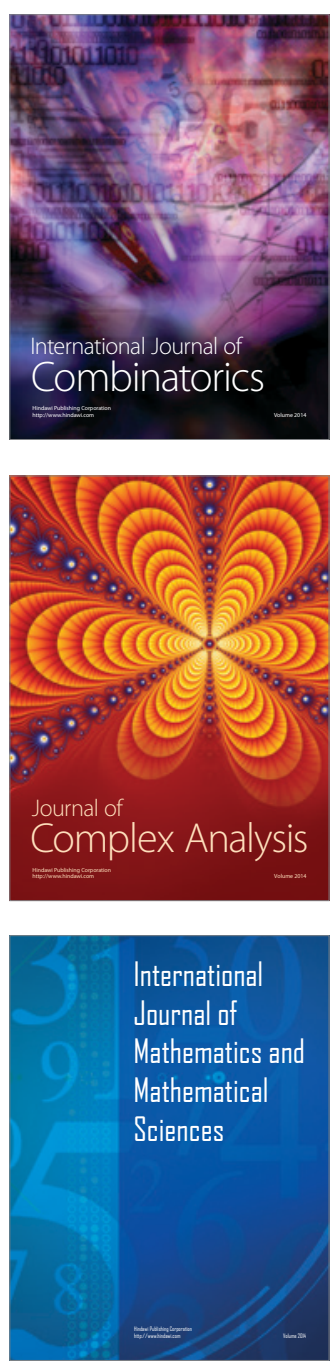
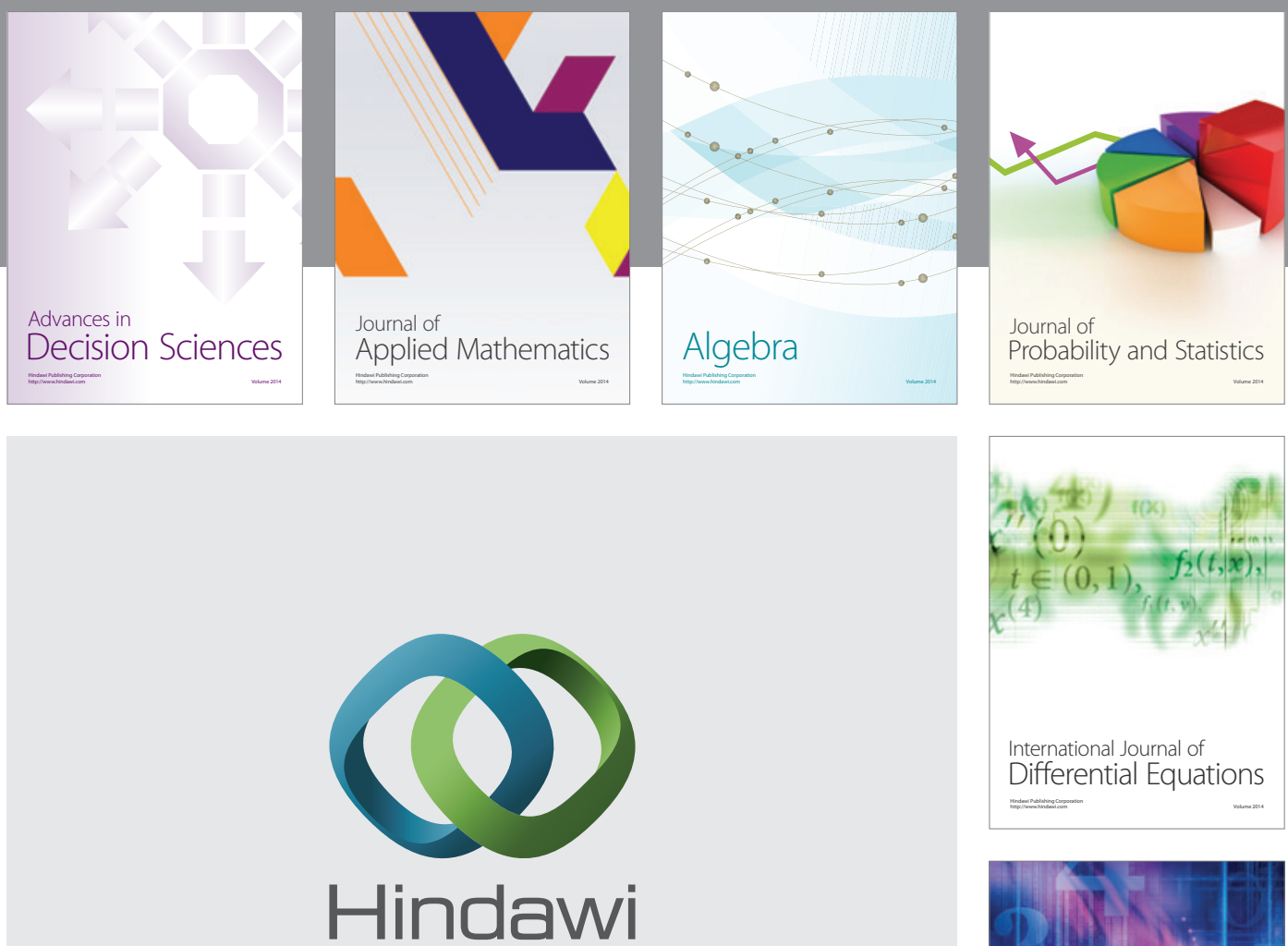

Submit your manuscripts at http://www.hindawi.com
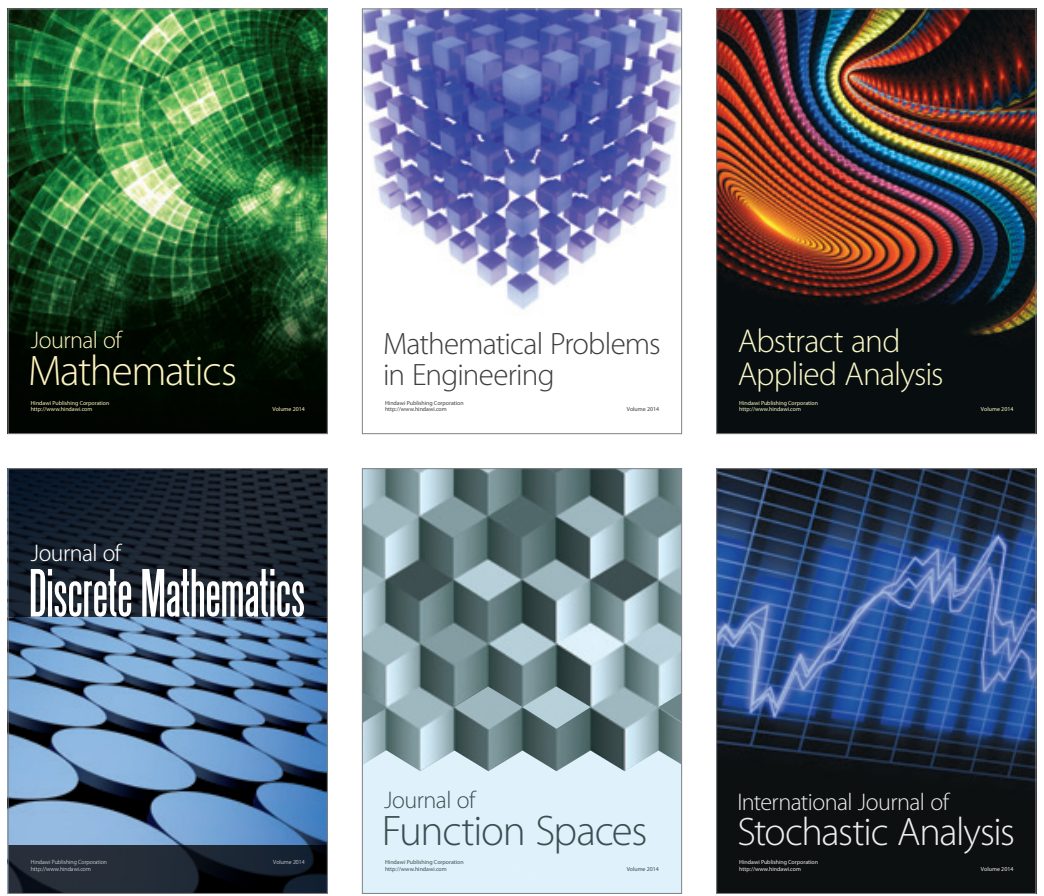

Journal of

Function Spaces

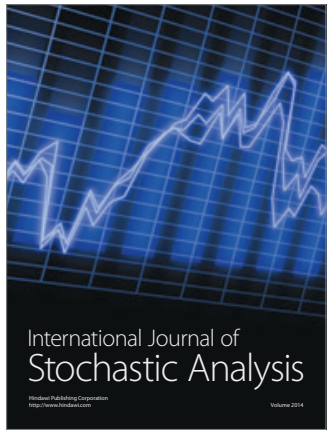

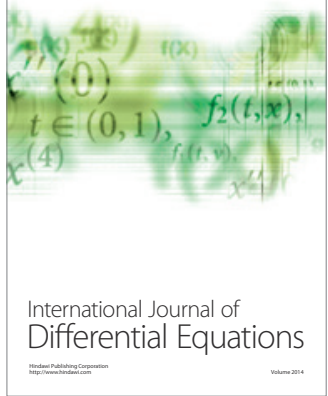
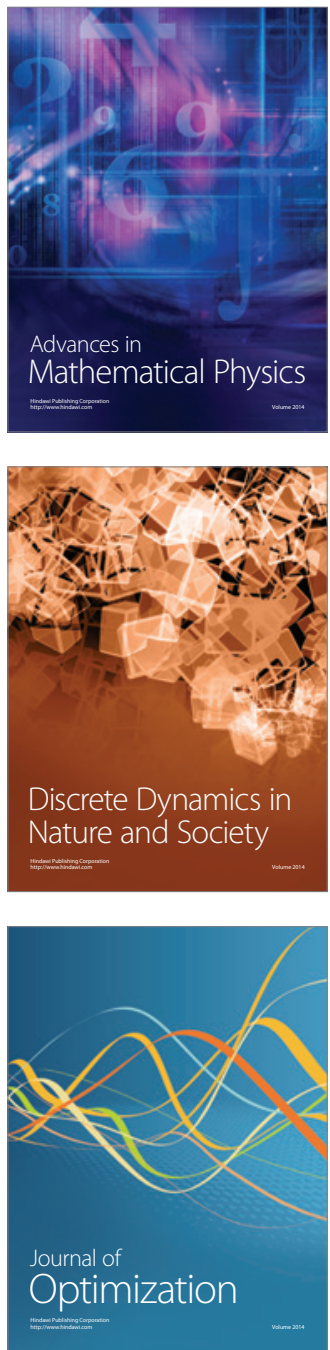